\title{
Lithium niobate transducers for MRI-guided
}

\section{ultrasonic microsurgery}

\author{
Spiros Kotopoulis ${ }^{1}$, Student Member, IEEE, Han Wang ${ }^{2}$, Sandy Cochran ${ }^{2}$, Member, IEEE, \\ and Michiel Postema ${ }^{1,3}$, Senior Member, IEEE \\ ${ }^{1}$ Department of Engineering, The University of Hull, Kingston upon Hull HU6 7RX, United Kingdom.
}

${ }^{2}$ Institute for Medical Science \& Technology, School of Engineering, Physics and Mathematics, University of Dundee, Dundee DD2 1FD, United Kingdom.

${ }^{3}$ Department of Physics and Technology, University of Bergen, Allégaten 55, 5007 Bergen, Norway.

\begin{abstract}
Focused ultrasound surgery (FUS) is usually based on frequencies below $5 \mathrm{MHz}$, typically around $1 \mathrm{MHz}$. Whilst this allows good penetration into tissue, it limits the minimum lesion dimensions that can be achieved. In the study reported here, we investigate devices to allow FUS at much higher frequencies, therefore in principle reducing the minimum lesion dimensions. Furthermore, FUS can produce deep-sub-millimetre demarcation between viable and necrosed tissue; high frequency devices may allow this to be exploited in superficial applications which may include dermatology, ophthalmology, treatment of the vascular system, and treatment of early dysplasia in epithelial tissue. In this paper we explain the methodology we have used to build high-frequency high-intensity transducers using Y-36 ${ }^{\circ}$ cut lithium niobate. This material was chosen as its low losses give it the potential to allow very high-frequency operation at harmonics of the fundamental operating frequency. A range of single element transducers with a centre frequency between $6.6 \mathrm{MHz}$ and $20.0 \mathrm{MHz}$ were built and the transducers' efficiency and acoustic power output were measured. A focused 6.6-MHz transducer was built with multiple elements operating together and tested using an ultrasound phantom and MRI scans. It was shown to increase phantom temperature by $32^{\circ} \mathrm{C}$ in a localised area of $2.5 \mathrm{~mm} \times 3.4 \mathrm{~mm}$ in the plane of the MRI scan. Tests on poultry were also performed and shown to create lesions of similar dimensions. This study therefore demonstrates that it is feasible to produce high-frequency transducers capable of high-resolution FUS using lithium niobate.
\end{abstract}




\section{Lithium niobate transducers for MRI-guided}

\section{ultrasonic microsurgery}

\section{INTRODUCTION}

Focused ultrasound surgery (FUS) is based on the application of high intensity focused ultrasound (HIFU) to heat tissue to a temperature that causes protein denaturation and coagulative necrosis [1]. The required temperature to generate lesions is between $56-60^{\circ} \mathrm{C}$ [2], [3]. The frequency of ultrasound used is generally around $1 \mathrm{MHz}$, generating characteristic ellipsoidal lesions on the order of $1 \mathrm{~cm}$ in length. Higher frequencies in the region of $4 \mathrm{MHz}$ are also used where more precise treatment is needed, for example in the prostate where tumour sizes may be $<1 \mathrm{~mm}$ long [4]. At such frequencies, conventional piezoelectric transducers can be used, based on hard piezoceramic with high drive capability. The use of FUS is increasing as a non-invasive form of surgery and the need for even higher precision is increasing for example for use in aesthetic facial rejuvenation [5], ultrasonic thrombolysis [6] and treatment of malignant disease in breast [7] whilst helping maintain a patient's quality of life when compared to invasive surgery [8].

In this paper, we consider the type of device that could be used to apply HIFU at much higher frequencies, with our research ultimately targeting $50-100 \mathrm{MHz}$. A difficulty with FUS is to necrose a clinically significant volume quickly enough for financial viability within modern medical treatment systems. However, the exquisite precision of necrosis possible with HIFU must also be recognised. The interface region may be as thin as just a few cells, with deep sub-millimetre dimensions, offering interesting possibilities for precise intervention. These may include treatment of dermatological and ophthalmological conditions, of the walls of the digestive system, for example relating to pre-cancerous tissue dysplasia, and of the walls of the vascular system. The superficial nature of these applications, if necessary handled with intralumenal and intravascular devices, significantly eases the problem of penetration depth at high frequency. The formats of the devices we report here are not suitable for these applications directly but reports on transurethral and endocavitary devices [9], [10] point the way forward. In all cases, we would predict adoption of high frequency FUS for superficial treatments of small overall volumes. As the attenuation coefficient of human tissue has a near linear dependence on frequency [11] greater intensity fields are necessary at higher frequencies in order to be able to penetrate deep enough into human tissue even for superficial applications. Piezoceramic is expected to be incapable of sustaining sufficiently high-power operation at such frequencies because of mechanical fragility, losses, and electrical breakdown. Instead, we have based our investigation on lithium niobate, $\mathrm{LiNbO}_{3}$ [12]. As a single crystal, this can be thinned easily without disintegrating 


\section{Methodology}

\section{A. Lithium niobate}

As it was expected that piezoceramics would be unable to produce HIFU at high frequencies and harmonics due to de-poling or cracking, we have explored $\mathrm{Y}-36^{\circ} \mathrm{LiNbO}_{3}$. As well as its basic advantages, it has a high resonant frequency of $3.3 \mathrm{MHz} \mathrm{mm}^{-1}$, thus allowing for thicker elements at higher frequencies for cost effective manufacturing compared to piezoceramics, and it has the highest electromechanical coupling coefficient compared to other $\mathrm{LiNbO}_{3}$ cuts [15].

\section{B. Transducer manufacture}

Three transducer designs were prepared as shown in Fig.1: unfocused single elements with $17 \mathrm{~mm}$ square $\mathrm{LiNbO}_{3}$ plates (xDucer 1); a 2D faceted bowl with three pentagonal and four hexagonal plates to mimic a spherically-focused device (xDucer 2); and a 1D faceted cylindrical section with five, $9 \times 30(\mathrm{~mm})^{2}$ rectangular plates to mimic a cylindrically focused device (xDucer3). The equivalent radii of curvature for xDucer 2 and xDucer 3 were $50 \mathrm{~mm}$ and $30 \mathrm{~mm}$ respectively. The devices were manufactured as prototypes using a proof-of-concept approach; this limited their reliability and hence sometimes the completeness of the data that was recorded. Nevertheless, the manufacturing techniques and the principles of the devices could be taken forward to more robust examples.

To prepare the plates for each transducer, Y-36 ${ }^{\circ}$ cut, 3-inch diameter, $0.5-\mathrm{mm}$ thick $\mathrm{LiNbO}_{3}$ wafers $($ Boston Piezo-Optics, Inc, Boston, MA) were obtained, polished on one side and lapped on the other. Figure 1 shows the position of each element from each wafer for each of the three transducer designs. Separation of the plates was performed with a programmable APD1 saw (Logitech Ltd, Glasgow, UK) with a spindle speed of $2900 \mathrm{rpm}$ and a feed rate of $0.160 \mathrm{~mm} \mathrm{~s}^{-1}$.

For the xDucer 1 devices, the 11 square elements cut from a single wafer were lapped individually in steps of $30 \mu \mathrm{m}$ starting from $500 \mu \mathrm{m}$ down to $200 \mu \mathrm{m}$ using a PM5 precision lapping and polishing machine (Logitech Ltd, Glasgow, UK). The force applied during lapping was adjusted depending on the sample size, typically in the range $400-900 \mathrm{~g}$. A slurry of $20-\mu \mathrm{m}$ calcined $\mathrm{Al}_{2} \mathrm{O}_{3}$ powder in water was used as abrasive. Once the elements reached within $25 \mu \mathrm{m}$ of the target thickness, 9- $\mu \mathrm{m}$ calcined $\mathrm{Al}_{2} \mathrm{O}_{3}$ powder was used to avoid scratching. The lapping machine was programmed to ensure maximum flatness.

The true thickness of the samples was measured and verified at regular intervals using a CG-10 Precision Electronic Measurement System (Logitech Ltd, Glasgow, UK). Once each element was flat at the desired thickness, 
it was removed from the glass lapping plate and re-measured to verify the thickness. The elements were continuously checked using a stereo microscope for flaws which could act to concentrate stress and lead to cracking.

An electrode was hand painted on to the lapped side using ELECTRODAG 1415 silver paint (Acheson Colloids BV, Scheemda, Netherlands). Excess paint around the edges was removed using a scalpel and acetone. The polished side of each element was then attached to the adhesive side of Adwill D-210 UV tape (Lintec of America, Inc., Phoenix, AZ). RG174A/U $50 \Omega$ coaxial cable was used connected to the plates with Ag-loaded conductive epoxy, curing taking place at $80^{\circ} \mathrm{C}$ for $10 \mathrm{~min}$.

For xDucer 1 devices, $\mathrm{Cu}$ tubing with an internal diameter of $28 \mathrm{~mm}$ was cut into lengths of $50 \mathrm{~mm}$ and placed over the $\mathrm{LiNbO}_{3}$ plates onto the adhesive side of the UV tape. Epoxy was then introduced around the sides of the $\mathrm{LiNbO}_{3}$ plate to join it to the $\mathrm{Cu}$ tube. The case for the 2D faceted array, xDucer 2, had a height of $75 \mathrm{~mm}$, outer diameter of $70 \mathrm{~mm}$ and a wall thickness of $2 \mathrm{~mm}$. The case for the curvilinear array, xDucer 3, had a height of $75 \mathrm{~mm}$, outer diameter of $50 \mathrm{~mm}$ and wall thickness of $1.5 \mathrm{~mm}$. For operation within a magnetic resonance imaging (MRI) system, the cases of xDucer 2, and 3 were polyvinyl chloride (PVC) coated with a thin layer of Ag paint so they could be used as the electrical ground connections to the front face of each transducer.

To support the fragile $\mathrm{LiNbO}_{3}$, Epofix resin (Struers, Ballerup, Denmark) was mixed with S38 glass microballoons (Lawrence Industries, Tamworth, UK) with a weight ratio of $65: 35$. The microballoon-epoxy mix was poured into the transducer shell. The xDucer 1 devices were filled to a depth of $16 \mathrm{~mm}$ whereas xDucers 2 and 3 were filled to a depth of $22 \mathrm{~mm}$. It was found that the acoustic output with the backing material was reduced by $5 \%$ compared to devices made without backing. The backing was left to cure at room temperature. The earth cable was attached to the shell using conductive Ag epoxy. The UV tape was then exposed to UV light and peeled off. Any remaining adhesive residue was removed manually.

The exposed $\mathrm{LiNbO}_{3}$ was cleaned using solvent then the front surfaces and part of each case were painted with Ag paint. The cases were then filled with 5368 silicon (Henkel AG \& Co. KGaA, Düsseldorf, Germany) to waterproof the cables and 50- $\Omega$ BNC RG-174 plugs were connected to the coaxial cables.

\section{Acoustic pressure}

Each transducer was driven by a continuous wave at its fundamental frequency, generated by an AFG3102 waveform generator (Tektronix, Everett, WA). The signal was passed through a $-20-\mathrm{dB}$ attenuator before being used as the input to a power amplifier. The single element transducers were tested using a $3100 \mathrm{LA},+55-\mathrm{dB}$ RF amplifier (Electronics \& Innovation, Rochester, NY). To test xDucer 2, the pentagonal elements were linked and driven by a $2100 \mathrm{~L},+50-\mathrm{dB}$ RF amplifier (Electronics \& Innovation, Rochester, NY) and the hexagonal elements were linked and driven by the 3100LA amplifier. This was done to give the ability to improve on the 
alignment of the multiple sound fields by shifting the phase of each group of elements. The pressures outputs were measured using a calibrated fibre-optic hydrophone (Precision Acoustics, Dorchester, UK) and verified using an HGL-0200 piezoelectric hydrophone (Onda, Sunnyvale, CA). The curvilinear transducer, (xDucer 3), was tested using a 150A250, 150W RF amplifier (Amplifier Research, Souderton, PA). The acoustic pressure was measured at the acoustic focus, $13 \mathrm{~mm}$ from the transducer face using the HGL-0200 hydrophone. The maximum peak-to-peak acoustic pressure was defined at the acoustic focus of each transducer in a $25 \times 17 \times 12(\mathrm{~cm})^{3}$, water filled, low-density polyethylene container. For all measurements the free field was manually scanned to locate the acoustic focus of each transducer using an M-652 $x-y-z$ micro-translation stage (Newport, Didcot, Oxfordshire, UK).

\section{D. $\mathrm{LiNbO}_{3}$ Properties}

Data available for the properties of $\mathrm{Y}-36^{\circ} \mathrm{LiNbO}_{3}$ were found to be limited and incomplete in the literature so values for one-dimensional simulation were obtained using PRAP version 2.2 software (TASI Technical Software Inc, Ontario, Canada) using electrical impedance data from a plate measured with a 4395A impedance analyser (Agilent, Santa Clara, CA). Table I shows the measured properties for $\mathrm{Y}^{-36^{\circ}}$ cut $\mathrm{LiNbO}_{3}$, with figures for Z-cut material shown for comparison. The resonance frequencies of the transducers were also measured using the same impedance analyser.

\section{E. Acoustic radiation}

The acoustic radiation force output of the transducers was measured using an EMS Model 67 ultrasound radiation force balance (EMS Physio Ltd, Wantage, UK). The transducers were placed within $20 \mathrm{~mm}$ of the surface of the ultrasound absorber in the balance to ensure that the total radiated flux was incident on it. The output voltage of the waveform generator was increased and the amplifier forward and reflected power and the transducer acoustic power were recorded.

\section{F. MRI temperature measurements}

MRI guidance is used for FUS [16] as it allows precise targeting of the HIFU field and direct temperature measurement at the focus. For MRI-guided focused ultrasound surgery (MRgFUS) tests in the present work, xDucer 3 and a DQA Gel Phantom (ATS Laboratories, Bridgeport, CT) placed in a cylindrical perspex chamber filled with tap water were placed in a GE Signa HDx 1.5T MRI system (GE Healthcare, Waukesha, WI). A gradient echo planar image (EPI) was recorded with $\mathrm{TE}=17.0 \mathrm{~ms}, \mathrm{ER}=230.0 \mathrm{~ms}$ and $\mathrm{BW}$ at $62.0 \mathrm{kHz}$ to capture the temperature increase of the phantom. The curvilinear transducer was turned on at $\mathrm{t}=0 \mathrm{~s}$ with a pk-pk input voltage of $101 \mathrm{~V}$, equivalent to $8 \mathrm{~W}$ acoustic power and $32 \mathrm{~W}$ forward electrical power. The transducer was turned off after $55 \mathrm{~s}$. The size of the acoustic focus was determined by the area heated above the surrounding ambient temperature. 


\section{G. Tissue sonication}

To test the effect of the HIFU field on tissue, two boneless, skinless chicken breasts (Tesco, Cheshunt, UK) were cut into $12,2 \times 2 \times 8(\mathrm{~cm})^{3}$ strips. The strips were placed in a $10 \times 15 \times 5(\mathrm{~cm})^{3}$ container filled with tap water at room temperature and xDucer 3 was clamped vertically with the acoustic focus on the surface of the tissue. Each sample was sonicated once. For tests beneath the surface of the tissue the transducer was lowered closer to the chicken. In all experiments it was ensured that the transducer surface was not in contact with the tissue. The chicken breast was sonicated using the same settings as in the MRgFUS measurements. Sonication time was increased in steps of $10 \mathrm{~s}$. Lesion sizes were measured manually using ImageJ (National Institutes of Health, Bethesda, MD).

\section{Results AND Discussion}

\section{A. $\mathrm{LiNbO}_{3}$ Properties}

The resonant frequency and third harmonic of the xDucer 1 devices made with different $\mathrm{LiNbO}_{3}$ thicknesses are compared to one-dimensional modelling (ODM) for both Z-cut and Y-36 $6^{\circ}$ cut $\mathrm{LiNbO}_{3}$ in Fig. 2. Although the Z-cut material gives a higher frequency for a given material thickness, other key properties such as $d_{33}$ and $k_{T}$ are much lower, hence the preference for $\mathrm{Y}-36^{\circ}$ cut material.

\section{B. Acoustic pressure}

The acoustic pressures generated by the three transducers is given in Table II. The xDucer 2 device generated a modulated sound field. It was possible to improve the output and reduce the envelope frequency of the modulation by shifting the phase of each set of elements. The lowest modulation frequency of $550 \mathrm{kHz}$ was achieved with a phase difference of $12^{\circ}$. The modulated sound field was generated as a result of multiple interacting sound fields. This was due to misaligned elements in our proof of concept devices. To cancel out the modulation, the phase of each element would have to be controlled independently, or the faceted bowl must be manufactured to tolerances

at $<\frac{1}{4} \lambda$ of the operating frequency. Higher harmonics of xDucer 2 were not tested due to low reproducibility. The acoustic pressures generated were limited by the maximum outputs of the RF amplifiers used. From the fundamental pressure measurements, the design of xDucer 2 would be the most logical to pursue.

\section{Acoustic Radiation Power}

The acoustic power generated by the xDucer 1 devices is shown in Fig. 3. Efficiency for these devices was found to be $33 \pm 5 \%$ throughout the frequency spectrum. Sustained operation of up to 5 minutes was possible without damaging the transducers at the resonance frequency, $3^{\text {rd }}$ and $5^{\text {th }}$ harmonic. The output power is seen to drop as the element thickness decreases. This is due to the increasing electrical impedance mismatch shown in Fig. 4 . For 
maximum output power the impedance magnitude and phase should be $50 \Omega$ and $0^{\circ}$ respectively. As the element thickness decreases the impedance magnitude also decreases. This is seen for both the fundamental resonance and $3^{\text {rd }}$ harmonic. The phase of the $3^{\text {rd }}$ harmonic increases with element thickness due to the inductance generated by the cable. Factors such as amount of Ag-loaded epoxy and cable length were seen to affect the impedance of the transducers [17]. The xDucer 3 device had an efficiency of $25 \pm 2 \%$ whereas a commercial $3.28-\mathrm{MHz}, 58-\mathrm{mm}$ diameter HIFU transducer made with piezoceramic (Precision Acoustics, Dorchester, UK) was found to have an efficiency of $20 \pm 1 \%$.

\section{MRI temperature measurements}

Figure 5 is an MRI image of xDucer 3 positioned on the DQA gel phantom. Figure 6 shows the area heated by xDucer 3 in the plane of the MRI scan, aligned with its focus. The surface area of heating after $55 \mathrm{~s}$ of sonication was $2.5 \mathrm{~mm} \times 3.4 \mathrm{~mm}$. Within $31 \mathrm{~s}$ the temperature in the acoustic focus of the transducer had increased $18^{\circ} \mathrm{C}$ above ambient to a temperature of $38^{\circ} \mathrm{C}$. A peak temperature of $52^{\circ} \mathrm{C}$ was reached after $55 \mathrm{~s}$ of sonication, $32^{\circ} \mathrm{C}$ above ambient, as shown in Fig. 7. The acoustic intensity at the focus of the transducer was equivalent to $163 \mathrm{Wcm}^{-2}$. After sonication, cavitation related bubbles formed on the front surface of the transducer shown in Fig. 5. These measurements demonstrate the viability of MRI ultrasonically-elevated temperature measurement with the devices reported here; it should be noted that at the $3^{\text {rd }}$ and $5^{\text {th }}$ harmonics $(21.1 \mathrm{MHz}$ and $35.2 \mathrm{MHz}$ respectively), the ultrasound wavelength is substantially smaller than the spatial resolution of the 1.5T MRI system.

\section{E. Tissue sonication}

Figure 8 shows the effect of xDucer 3 on chicken breast. An increased sonication time was necessary in order to induce a lesion beneath the tissue surface, of similar size to the MRI measured results. This is partially due to scattering and gas content in the tissue. The lesions dimensions after $90 \mathrm{~s}$ of sonication at $6.6 \mathrm{MHz}$ matched those measured with the MRI. In Fig. 8, the chicken has been sliced open to locate the region of sonication. The lesion was formed $60 \mathrm{~mm}$ beneath the surface of the tissue without affecting the upper tissue boundary or the surrounding tissue. In further tests, after two minutes continuous sonication, when the transducer surface was in contact with the chicken tissue, sufficient heat was generated to cause protein denaturation on the chicken surface. Thus, in a practical (clinical) setting, contact with tissue would have to be avoided. Impedance matching and better-attached electrodes might reduce element heating; water-cooling of commercial FUS transducers is also common.

\section{CONCLusions}

In conclusion, we have shown that it is feasible to manufacture high-frequency, high-intensity, focused ultrasound transducers based on $\mathrm{Y}-36^{\circ}$ cut $\mathrm{LiNbO}_{3}$. In a range of tests, we have demonstrated operating frequencies up to 
more than $50 \mathrm{MHz}$ using the $3^{\text {rd }}$ harmonic of $200-\mu \mathrm{m}$ thick $\mathrm{LiNbO}_{3}$, focal pressures of $4 \mathrm{MPa}$ at $35 \mathrm{MHz}$, and $\mathrm{MI}=4.7$ at $6.6 \mathrm{MHz}$. Two of the devices made, with faceted bowl and faceted cylindrical sections respectively, were designed to be operated under MRI guidance. We have shown that this design was successful and have used one of the devices to increase the temperature within a gel phantom, measured with MRI, to more than $50^{\circ} \mathrm{C}$ following sonication of $55 \mathrm{~s}$ with an equivalent acoustic intensity of $163 \mathrm{Wcm}^{-2}$. We also created lesions within chicken tissue after 90 s sonication.

Several aspects can be addressed in order to improve the performance of the transducers. At high acoustic intensities the Ag-paint electrode was damaged. This is attributed to air pockets trapped between the electrode and the $\mathrm{LiNbO}_{3}$. The use of thin film $\mathrm{Cr}-\mathrm{Au}$, Ti-Pt or Al electrodes would be better acoustically and electrically as electrodes compared to conductive Ag paint [18]. The cases of the devices for MRI guidance were made with PVC tubing coated with Ag paint; using an alternative such as Cu-epoxy composite [19] would aid manufacture and reliability and assist with shielding. The thin $\mathrm{LiNbO}_{3}$ piezoelectric elements were supported by microballoon-filled epoxy backing; this reduced the transducer output thus necessitating exploration of support materials with a lower acoustic impedance or other methods to support the plates. Finally, electrical impedance matching was neglected. However, as frequency increases electrical impedance decreases and sustained operation would be enhanced by electrical impedance matching.

\section{ACKNOWLEDGMENT}

This work has been supported by UK EPSRC Grants EP/F037025/1 and EP/G01213X/1, DFG Emmy Noether Programme Grant 38355133, and HERI Research Pump Priming Fund. The authors would like to thank Logitech Ltd (Glasgow, United Kingdom) for the lapping/polishing and dicing equipment and Alex Volovick of InSightec Ltd. (Haifa, Israel) for assistance with the MRI measurements. 


\section{REFERENCES}

[1] J. Wu and W. Nyborg, Eds., Emerging Therapeutic Ultrasound. Hackensack: World Scientific, 2006.

[2] C. Hill and G. R. ter Haar, "Review article: High intensity focused ultrasound: Potential for cancer treatment." Brit. J. Radiol., vol. 68, pp. 1296-1303, 1995.

[3] G. R. ter Haar, "Ultrasound focal beam surgery," Ultrasound Med. Biol., vol. 21, pp. 1089-1100, 1995.

[4] O. Rouvière, F. Mège-Lechevallier, J.-Y. Chapelon, A. Gelet, R. Bouvier, F. Boutitle, and D. Lyonnet, "Evaluation of color doppler in guiding prostate biopsy after HIFU ablation," Eur. Urology, vol. 50, no. 3, pp. 490-497, 2006.

[5] W. White, I. Makin, P. Barthe, M. Slayton, and R. Gliklich, "Selective creation of thermal injury zone in the superficial musculoaponeurotic system using intense ultrasound therapy," Arch. Facial Plast. Surg, vol. 9, pp. 22-29, 2007.

[6] K. Tachibana and S. Tachibana, "Albumin microbubble echo-contrast material as an enhancer for ultrasound accelerated thrombolysis," Circulation, vol. 92, no. 5, pp. 1148-1150, 1995.

[7] T. Huston and R. Simmons, "Ablative therapies for the treatment of malignant diseases of the breast," Am. J. Surg, vol. 189, no. 6, pp. 694-701, 2005.

[8] J. J. M. C. H. de la Rosette, V. Mouraviev, and T. Polascik, "Focal targeted therapy will be a future treatment modality for early stage prostate cancer," Eur. Urology, vol. 8, pp. 424-432, 2009.

[9] R. Seip, M. Biswas, M. Kuznetsov, N. Sanghvi, T. Gardner, and N. Dabhoiwala, "Transurethral high intensity focused ultrasound: catheter based prototypes and experimental results," Proc. IEEE Ultrason. Symp., vol. 2, pp. 1413-1416, 2000.

[10] I. Wharton, I. Rivens, G. R. ter Haar, D. Gilderdale, D. Collins, J. Hand, P. Abel, and N. de Souza, "Design and development of a prototype endocavitary probe for high-intensity focused ultrasound delivery with integrated magnetic resonance imaging," J. Mag. Res. Imag., vol. 25, no. 3, pp. 548-556, 2007.

[11] M. Lyons and J. Parker, "Absorbtion and attenuation in soft tissues ii- experimental results," IEEE Trans. Ultrason., Ferroelect., Freq. Contr., vol. 35, no. 4, pp. 511-521, 1988.

[12] R. Weis and T. Gaylord, "Lithium niobate: Summary of physical properties and crystal structure," Appl. Phys. A, vol. 37, pp. 191-203, 1985.

[13] K. Snook, J.-Z. Zhao, C. Alves, J. Cannata, W.-H. Chen, R. Meyer, T. Ritter, and K. Shung, "Design, fabrication, and evaluation of high frequency, single-element transducers incorporating different materials," IEEE Trans. Ultrason., Ferroelect., Freq. Contr., vol. 49, no. 02, pp. 169-176, 2002.

[14] J. Cannata, T. Ritter, W.-H. Chen, R. Silverman, and K. Shung, "Design of efficient, broadband single-element (20-80 MHz) ultrasonic transducers for medical imaging applications," IEEE Trans. Ultrason., Ferroelect., Freq. Contr., vol. 50, no. 11, pp. 1548-1557, 2003.

[15] A. W. Warner, M. Onoe, and G. Coquin, "Determination of elastic and piezoelectric constants for crystals in class (3m)," J. Acoust. Soc. Am., vol. 42, no. 6, pp. 1223-1231, 1968.

[16] K. Hynynen, "Mri-guided focused ultrasound treatements," Ultrasonics, vol. 50, no. 2, pp. 221-229, 2010.

[17] L. Schmerr, A. Lopez-Sanchez, and R. Huang, "Complete ultrasonic transducer characterization and its use for models and measurements," Ultrasonics, vol. 44, no. 1, pp. e753-e757, 2006.

[18] M. Nakazawa, T. Kosugi, K. Nakamura, S. Ueha, A. Maezawa, and Y. Hirao, "A high frequency variable focus ultrasonic transducer using polyurea thin film," Proc. IEEE Ultrason. Symp., vol. 4, pp. 2313-2316, 2005.

[19] B. Gerold, S. Reynolds, A. Melzer, and S. Cochran, "Early exploration of MRI-compatible diagnositc ultrasound transducers," Proc. IEEE Int. Ultrason. Symp., 2010, in press. 


\section{LIST OF FIGURES}

Transducer manufacturing: (a) position of plates in single wafer used for each transducer, (b) dimensions of plates for each transducer, (c) computer-aided design representation of transducers, and (d)

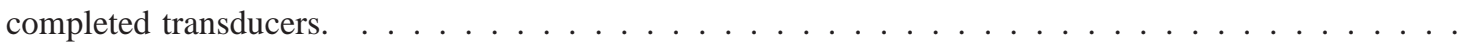

Resonance frequency and third harmonic as a function of element thickness for single-element $\mathrm{LiNbO}_{3}$ microballoon-backed transducers. The diamonds indicate the z-cut $\mathrm{LiNbO}_{3}$ simulations, the circles indicate the experimental measurements, and the five-point stars indicate the $\mathrm{Y}-36^{\circ} \mathrm{LiNbO}_{3}$ cut simulations. The grey data points show the $3^{\text {rd }}$ harmonic. The error bars indicate the confidence

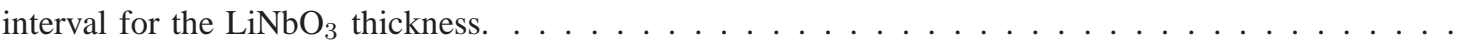
Acoustic power as a function of transducer input voltage for $17 \times 17(\mathrm{~mm})^{2}$ single element $\mathrm{LiNbO}_{3}$

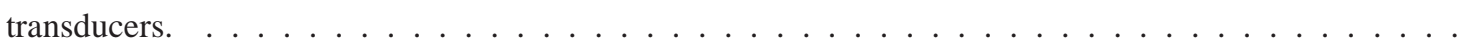
Minimum impedance magnitude and equivalent phase as a function of element thickness for single-element $\mathrm{LiNbO}_{3}$ microballoon backed transducers. The black diamonds indicate the impedance magnitude whereas the grey circles indicate the phase $\ldots \ldots \ldots \ldots \ldots$ (a) MRI side view of sonication setup where brightness indicates water content. Image is rotated $90^{\circ}$ anticlockwise from true position. (b) Schematic representation of sonication setup (i) perspex water bath, (ii) xDucer3, (iii) water, (iv) DQA gel phantom. The transducer generates negligible artefacts in the MRI image. The minor artefacts generated by the silver paint and coaxial cable are not in the acoustic path and thus do not affect the image or temperature measurement. . . . . . . . . . MRI view of sonication area. The focal region had a size of $2.5 \times 3.4(\mathrm{~mm})^{2}$. The green areas represents pixels of equal temperature, the blue areas represent the acoustic field, whereas the red areas represent pixels of temperature $>70^{\circ} \mathrm{C}$. The orange cross indicates the temperature measurement marker. . . Temperature increase as a function of time for xDucer 3 at the acoustic focus in ultrasonic phantom measured using MRI. The black diamonds indicates the temperature of the selected pixel, whereas the grey circles indicate average temperature of the eight surrounding pixels. . . . . . . . . Photograph showing lesion formed on chicken tissue after 90 s sonication using xDucer 3 . The acoustic focus was beneath tissue surface. The direction of acoustic propagation is into the image as indicated

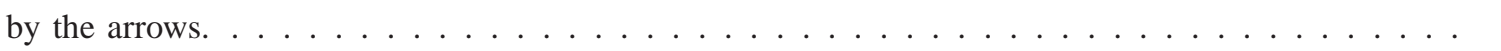


293 I Mechanical and piezoelectric properties for lithium niobate $\ldots \ldots \ldots \ldots \ldots \ldots$

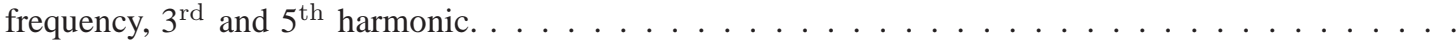




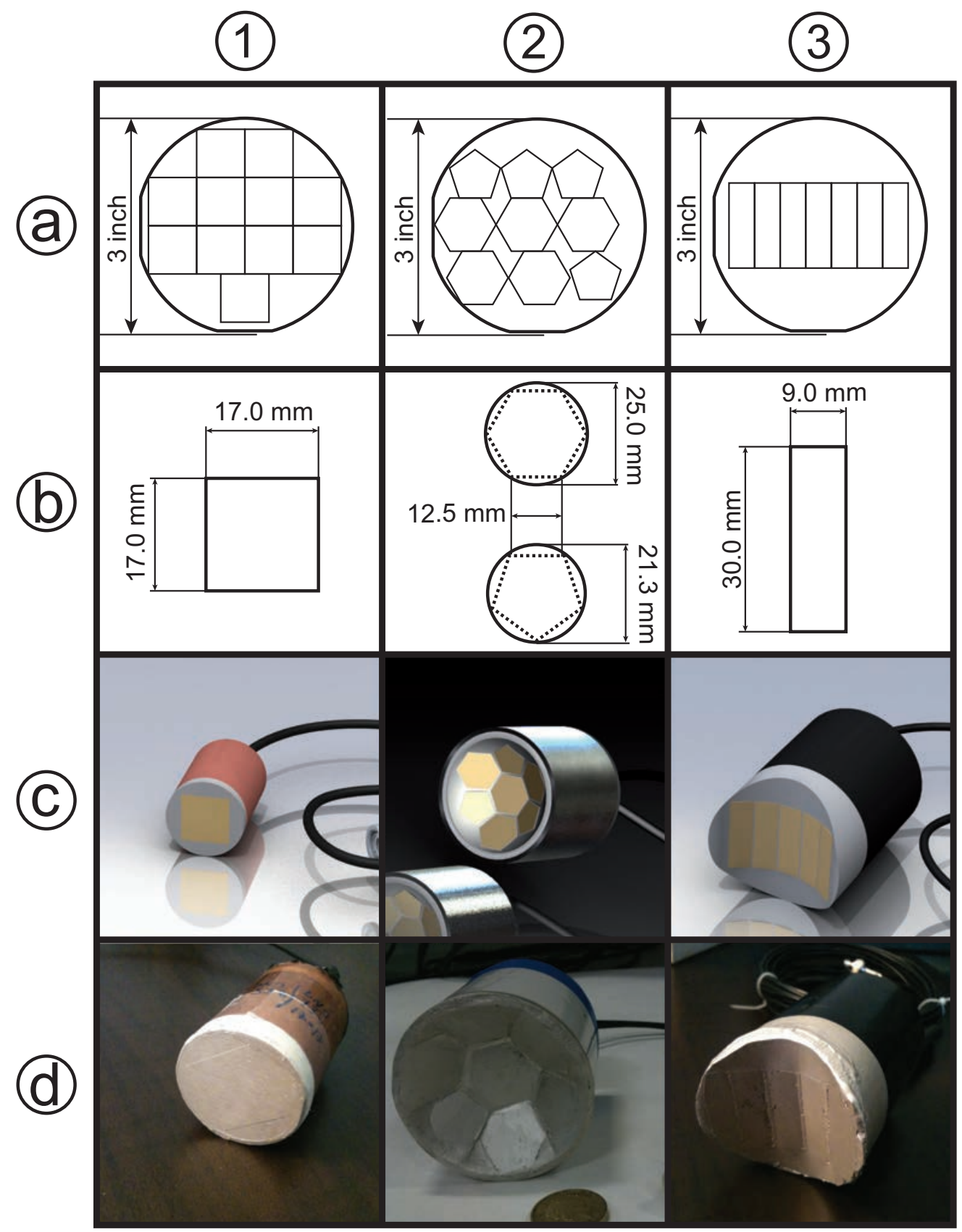

Fig. 1. Transducer manufacturing: (a) position of plates in single wafer used for each transducer, (b) dimensions of plates for each transducer, (c) computer-aided design representation of transducers, and (d) completed transducers. 


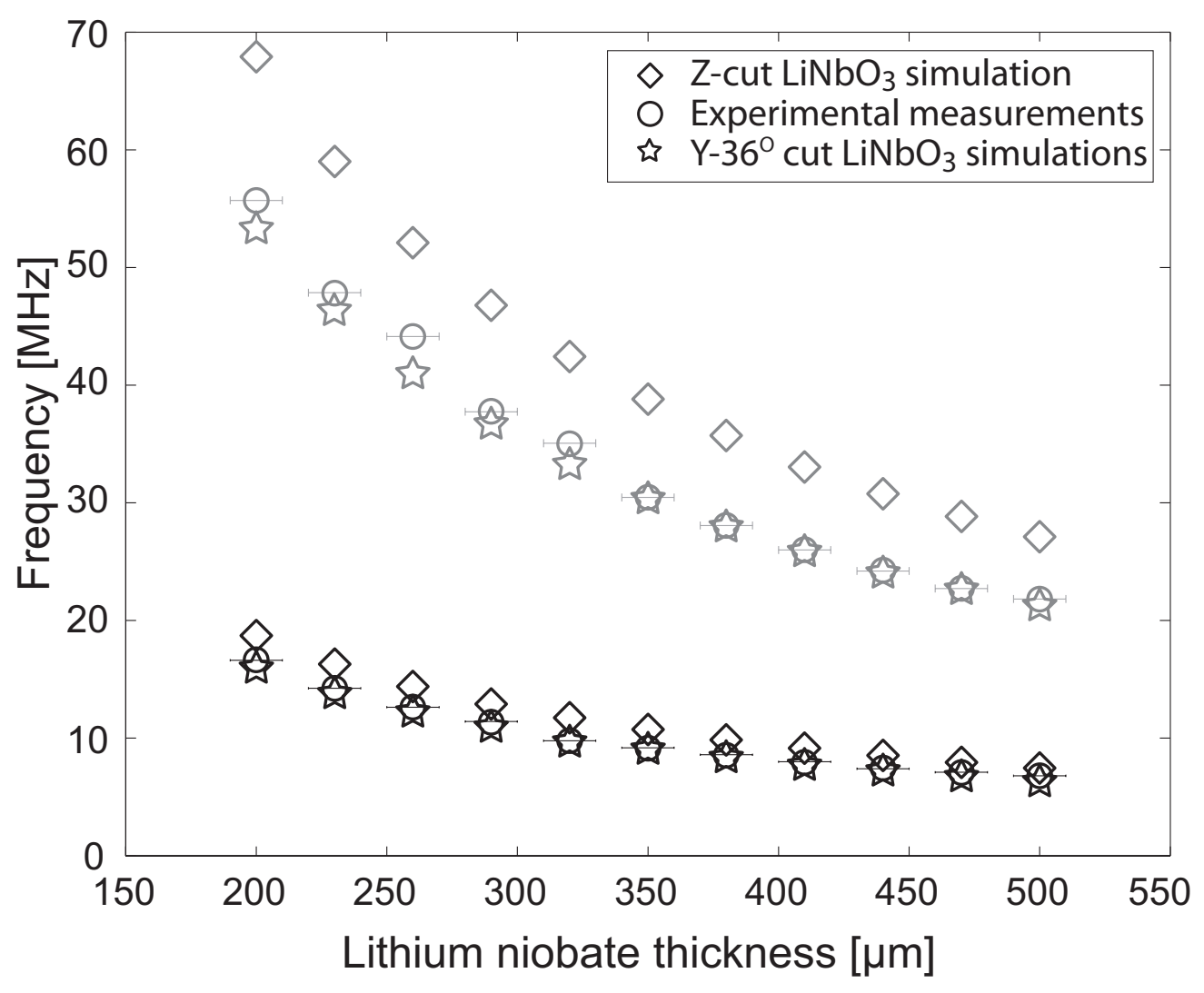

Fig. 2. Resonance frequency and third harmonic as a function of element thickness for single-element $\mathrm{LiNbO}_{3}$ microballoon-backed transducers. The diamonds indicate the $\mathrm{z}$-cut $\mathrm{LiNbO}_{3}$ simulations, the circles indicate the experimental measurements, and the five-point stars indicate the $\mathrm{Y}-36^{\circ} \mathrm{LiNbO}_{3}$ cut simulations. The grey data points show the $3^{\text {rd }}$ harmonic. The error bars indicate the confidence interval for the $\mathrm{LiNbO}_{3}$ thickness. 


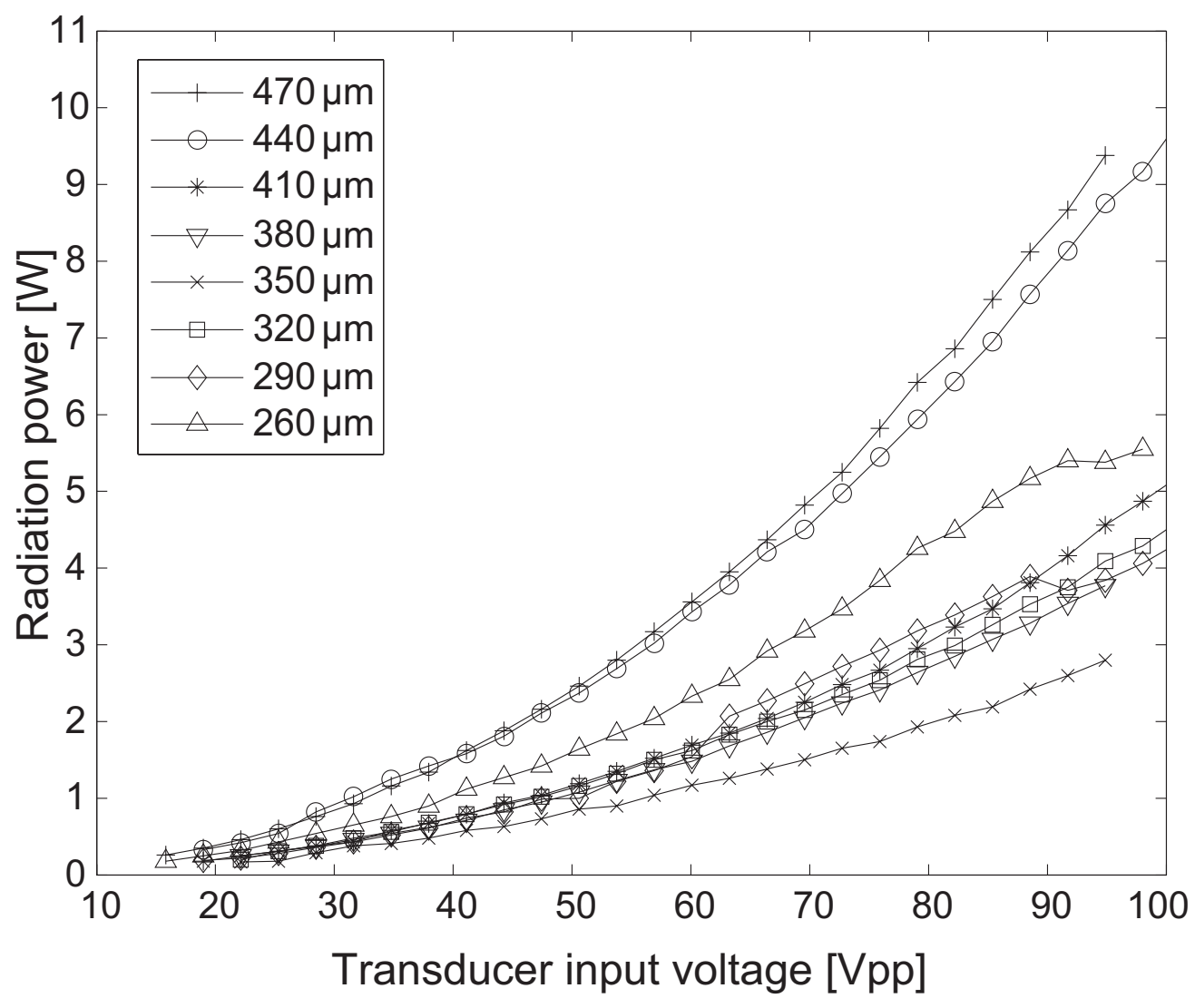

Fig. 3. Acoustic power as a function of transducer input voltage for $17 \times 17(\mathrm{~mm})^{2}$ single element $\mathrm{LiNbO}_{3}$ transducers. 

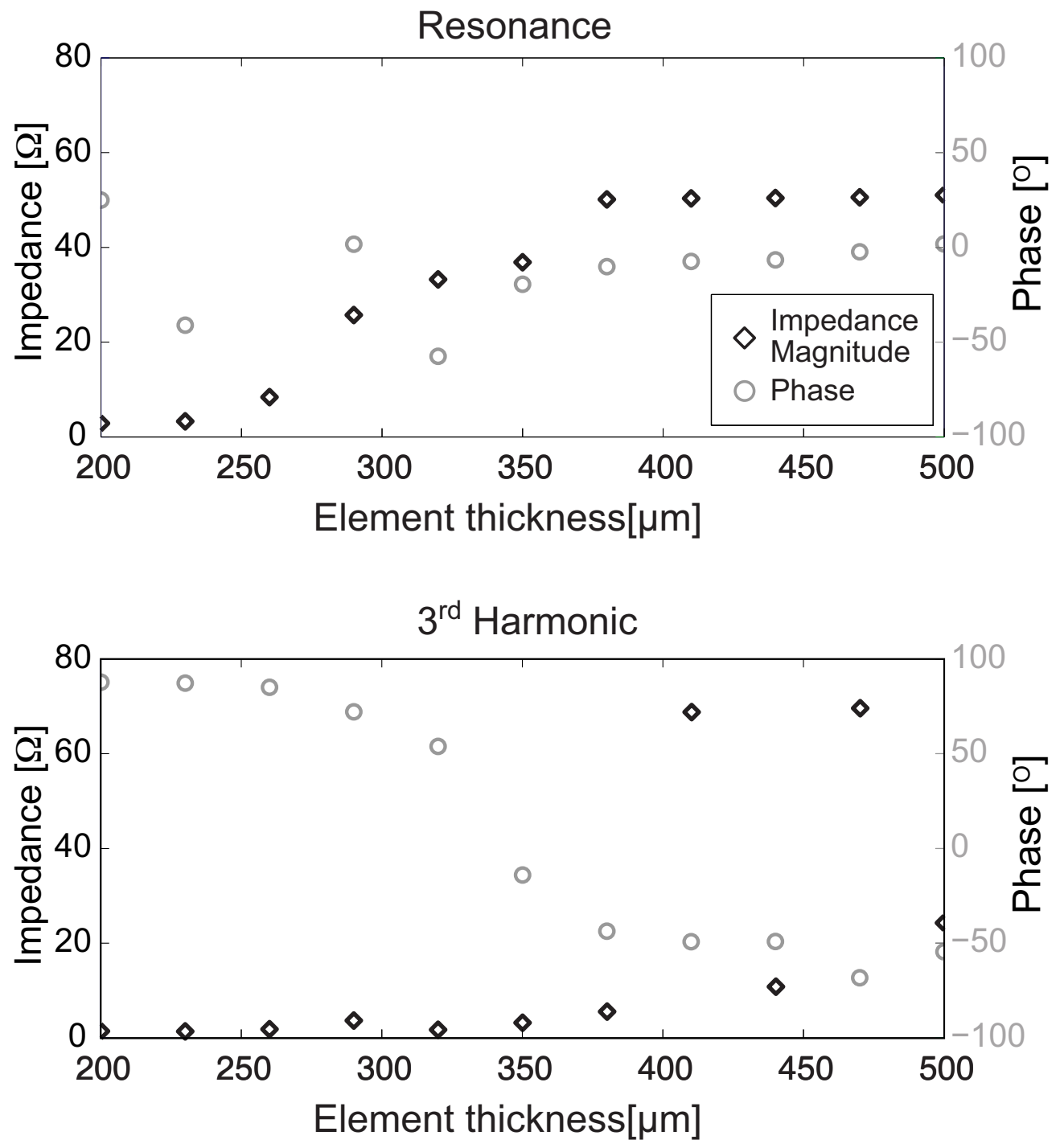

Fig. 4. Minimum impedance magnitude and equivalent phase as a function of element thickness for single-element $\mathrm{LiNbO}_{3}$ microballoon backed transducers. The black diamonds indicate the impedance magnitude whereas the grey circles indicate the phase. 


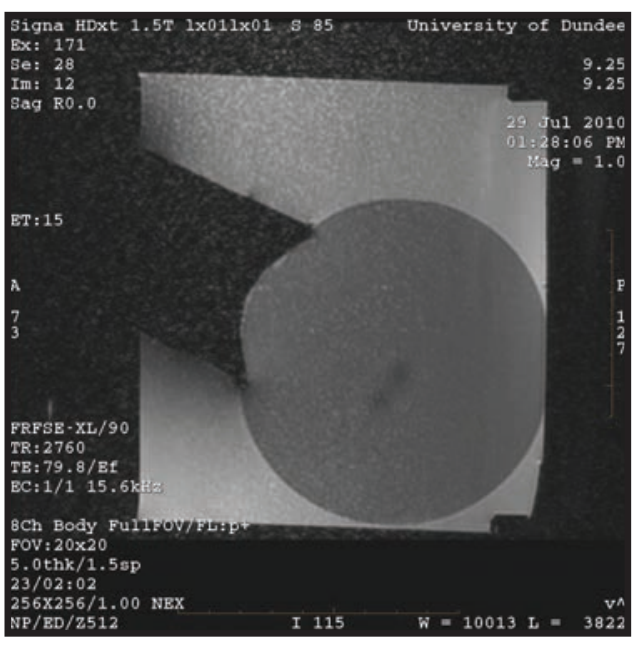

(a)

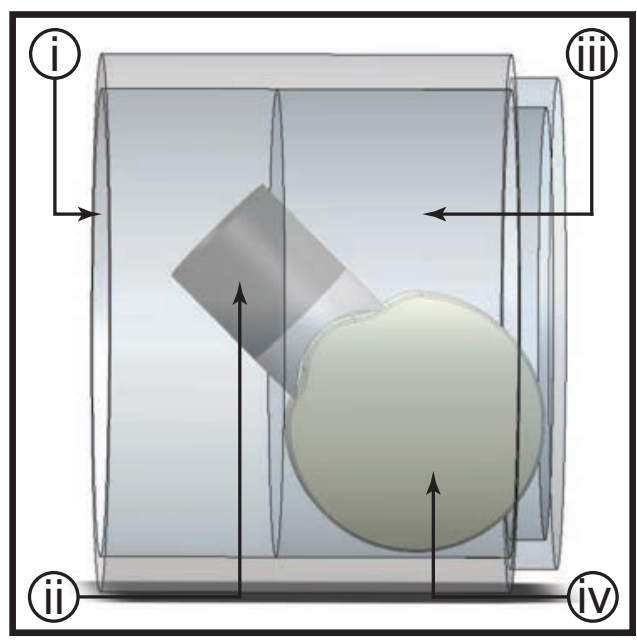

(b)

Fig. 5. (a) MRI side view of sonication setup where brightness indicates water content. Image is rotated $90^{\circ}$ anticlockwise from true position. (b) Schematic representation of sonication setup (i) perspex water bath, (ii) xDucer 3, (iii) water, (iv) DQA gel phantom. The transducer generates negligible artefacts in the MRI image. The minor artefacts generated by the silver paint and coaxial cable are not in the acoustic path and thus do not affect the image or temperature measurement. 


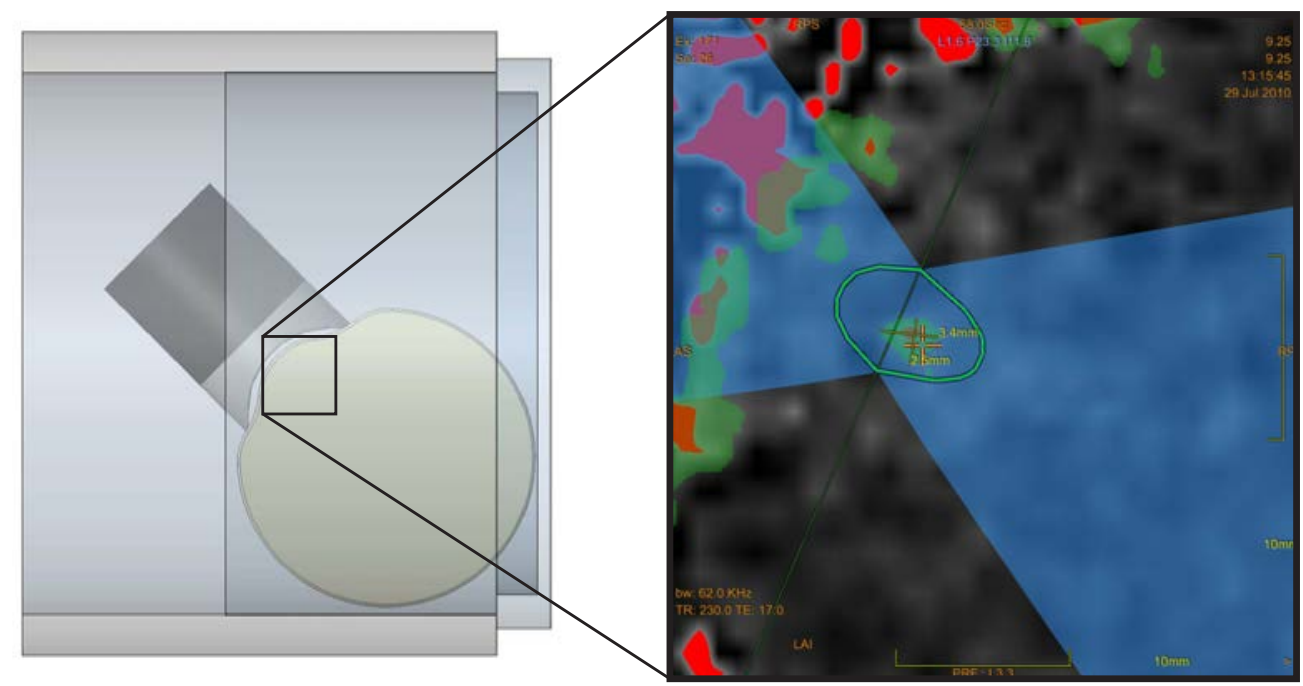

Fig. 6. MRI view of sonication area. The focal region had a size of $2.5 \times 3.4(\mathrm{~mm})^{2}$. The green areas represents pixels of equal temperature, the blue areas represent the acoustic field, whereas the red areas represent pixels of temperature $>70^{\circ} \mathrm{C}$. The orange cross indicates the temperature measurement marker. 


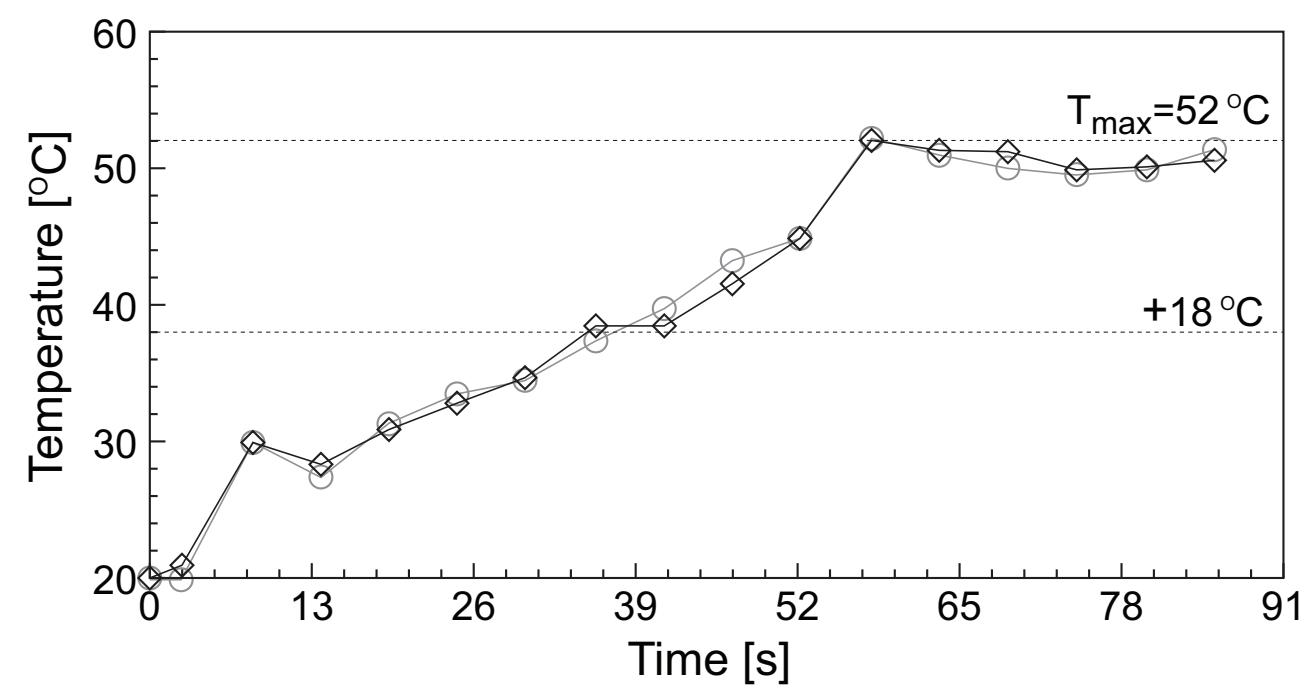

Fig. 7. Temperature increase as a function of time for xDucer 3 at the acoustic focus in ultrasonic phantom measured using MRI. The black diamonds indicates the temperature of the selected pixel, whereas the grey circles indicate average temperature of the eight surrounding pixels. 


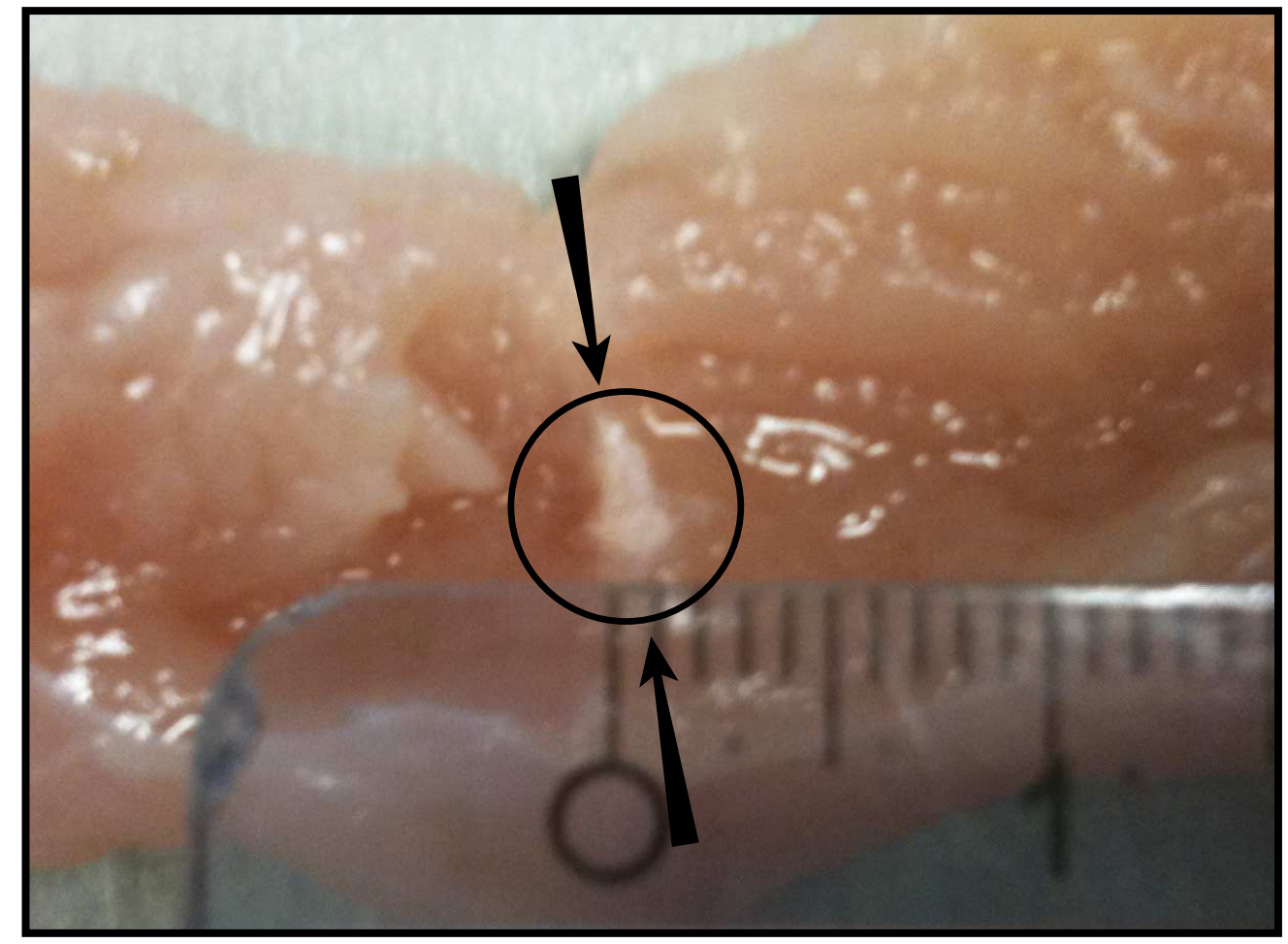

Fig. 8. Photograph showing lesion formed on chicken tissue after $90 \mathrm{~s}$ sonication using xDucer 3 . The acoustic focus was beneath tissue surface. The direction of acoustic propagation is into the image as indicated by the arrows. 


\begin{tabular}{|c|c|c|c|}
\hline Property & Parameter (unit) & Z-cut & Y-36 ${ }^{\circ}$ cut \\
\hline Density & $\rho\left(\mathrm{kg} \mathrm{m}^{-3}\right)$ & 4650 & 4650 \\
\hline Thickness mode velocity & $v\left(\mathrm{~m} \mathrm{~s}^{-1}\right)$ & 7380 & 7260 \\
\hline Acoustic impedance & $Z$ (MRayl) & 34.2 & 33.8 \\
\hline \multirow{3}{*}{ Elastic constants } & $c_{11}^{E}\left(\mathrm{Nm}^{-2}\right) \times 10^{9}$ & 203 & 185 \\
\hline & $c_{33}^{E}\left(\mathrm{Nm}^{-2}\right) \times 10^{9}$ & 245 & 185 \\
\hline & $c_{33}^{D}\left(\mathrm{Nm}^{-2}\right) \times 10^{9}$ & 252 & 245 \\
\hline \multirow{2}{*}{ Dielectric constants } & $\epsilon_{33}^{T} / \epsilon_{0}$ & 29.8 & 41.9 \\
\hline & $\epsilon_{33}^{S} / \epsilon_{0}$ & 25.7 & 37.6 \\
\hline \multirow{3}{*}{ Piezoelectric constants } & $e_{33}\left(\mathrm{Cm}^{-2}\right)$ & 1.3 & 4.47 \\
\hline & $h_{33}\left(\mathrm{~V} \mathrm{~m}^{-1}\right) \times 10^{9}$ & 5.71 & 13.4 \\
\hline & $d_{33}\left(\mathrm{~m} \mathrm{~V}^{-1}\right) \times 10^{-12}$ & 5.15 & 18.2 \\
\hline Electromechanical coupling coefficient & $k_{T}$ & 0.171 & 0.495 \\
\hline
\end{tabular}

TABLE I

MECHANICAL AND PIEZOELECTRIC PROPERTIES FOR LITHIUM NIOBATE 


\begin{tabular}{|c|c|c|c|c|c|c|}
\hline & \multirow[b]{2}{*}{ Frequency (MHz) } & \multirow[b]{2}{*}{ Wavelength $(\mu \mathrm{m})$} & \multicolumn{4}{|c|}{$\begin{array}{l}\text { Peak-to-peak acoustic pressure (MPa) } \\
\text { Equivalent mechanical index }[\mathrm{MI}]\end{array}$} \\
\hline & & & xDucer 1 & xDucer 2 & $\mathbf{x D}$ & $\operatorname{cer} 3$ \\
\hline Fundamental frequency & 6.6 & 226 & 14.1 & 24.3 & 16.7 & [3.3] \\
\hline $3^{\text {rd }}$ harmonic & 21.1 & 77 & {$[0.7]$} & - & 10.5 & [1.1] \\
\hline $5^{\text {th }}$ harmonic & 35.2 & 44 & {$[0.4]$} & - & 5.4 & [0.5] \\
\hline
\end{tabular}

TABLE II

PEAK-TO-PEAK ACOUSTIC PRESSURES GENERATED BY XDUCER 1, XDUCER 2 AND XDUCER 3 AT THEIR FUNDAMENTAL FREQUENCY, $3^{\text {rd }}$ AND $5^{\text {th }}$ HARMONIC. 


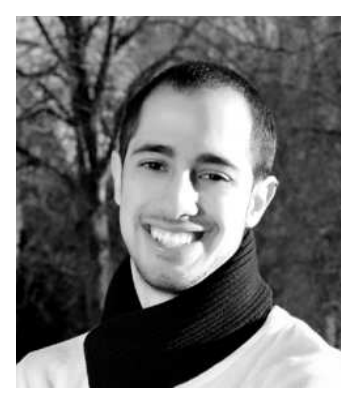

Spiros Kotopoulis (S’08) was born in Athens, Greece, in 1987. He received a first class B.Eng. (Hons) in Mechanical Engineering from The University of Hull, England, in 2008. Mr. Kotopoulis is a member of the U.K. Institute of Acoustics. He is currently pursuing a Ph.D. under the supervision of Professor Dr. Michiel Postema at The University of Hull. His research interests include high-speed photography, microscopy, ultrasound transducer manufacture and sonoporation.

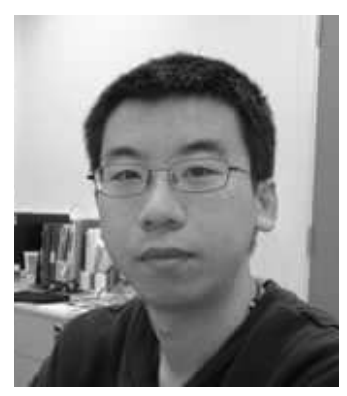

Han Wang was born in Tianjin, China, in 1986. He received his B.Eng. in Electronic Science and Technology from Tianjin University, China, in 2009. He then gained an MSc with Distinction in Biomedical Engineering from University of Dundee, Scotland, UK, in 2010. Mr. Han Wang is currently pursuing a Ph.D. under the supervision of Dr. Christine E. M. Démoré at Institute for Medical Science and Technology, University of Dundee. His current research interests are in ultrasonic device development for life sciences and electronics instrumentation for ultrasound devices and system. 


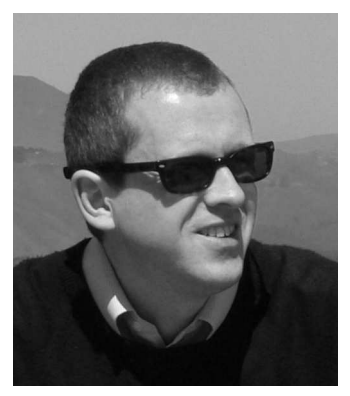

Sandy Cochran is Professor of Biophysical Science and Engineering, Deputy Director and Team Leader of Medical Ultrasound in the Institute for Medical Science and Technology, University of Dundee, Scotland. He received the B.Sc. degree in electronics and computing in 1986, the Ph.D. degree for work on ultrasonic arrays in 1990, and the MBA for an investigation of universities as part of an enterprise network in 2001, all from the University of Strathclyde. His present research interests are focused on medical ultrasound devices, with applications in diagnosis, image-guidance and therapy. He also maintains interests in relevant materials, systems design and applications issues, and in underwater sonar and industrial processing for medical and life sciences applications. He has worked extensively with industry internationally and collaborates with several academic research groups. His research income since 2009 as Principal Investigator has totalled more than $\$ 4.5 \mathrm{M}$. Outside work, he divides his time between his homes in Dundee and Glasgow.

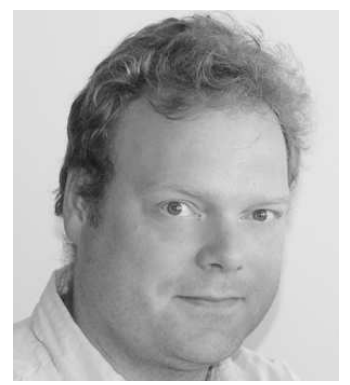

Michiel Postema (A'01-S'02-M'05-SM'08) was born in Brederwiede, Netherlands, in 1973. He received an M.Sc. in Geophysics from Utrecht University, Netherlands, in 1996 and a Doctorate in the Physics of Fluids from the University of Twente, Enschede, Netherlands, in 2004. Following a postdoctoral position at Ruhr-Universität Bochum, Germany, between 2005 and 2007, he became Lecturer in Engineering at The University of Hull, England. He was granted an Emmy Noether Research Group at Ruhr-Universität Bochum in 2009 and a Visiting Professorship at the University of Orléans, France, in 2010. In the same year, he obtained the Chair in Experimental Acoustics at the University of Bergen, Norway. Professor Dr. Postema is Associate Editor of Applied Acoustics (Elsevier) and 
member of the editorial board of Bubble Science, Engineering and Technology (Maney). He is also Fellow of the UK Institute of Acoustics (IOA), member of the IOA Research Coordination Committee and member of the Scientific Committee of Revue des Sciences et Technologie (Université de Batna). He has written more than 70 scientific publications on medical acoustics and cavitation, including 40 first-author papers and five co-authored textbooks. His particular expertise lies in analysing medical microbubble behaviour under sonication and in high-speed photography. He also explores non-medical applications of bubbles and droplets in sound fields. Since 2007, he has pulled in more than US\$4.4 Million in research grants. 